\title{
RELACIÓN ENTRE LOS COMPONENTES ÓPTICOS OCULARES E IMPLICACIONES EN EL PROCESO DE EMETROPIZACIÓN
}

\author{
THE RELATIONSHIPS BETWEEN OCULAR OPTICAL \\ COMPONENTS AND IMPLICATIONS IN THE PROCESS OF \\ EMMETROPIZATION
}

\author{
YEBRA-PIMENTEL E ${ }^{1}$, GONZÁLEZ-MÉIJOME JM² ${ }^{2}$ GARCÍA-RESÚA C ${ }^{3}$, \\ GIRÁLDEZ-FERNÁNDEZ $\mathrm{MJ}^{3}$
}

\section{RESUMEN}

Objetivo: Determinar la relación entre los diferentes componentes ópticos oculares y la emetropización en función de la longitud axial (LA).

Métodos: Participaron 109 jóvenes universitarios divididos en cinco grupos según el error refractivo: emétropes, hipermétropes, miopes bajos, miopes moderados y miopes altos. La medida de los parámetros intraoculares y el análisis de la topografía corneal se realizó mediante ultrasonografía y videoqueratoscopia respectivamente.

Resultados: Se encontró una correlación estadísticamente significativa entre la profundidad de la cámara anterior (PCA) y la LA en ojos con LA menor de $24 \mathrm{~mm}(r=0,441 ; p<0,001)$. Sin embargo en ojos con LA mayor no se encontró tal relación $(r=0,098 ; p=0,527)$. El espesor del cristalino (EC) mostró una correlación inversa con la LA para los ojos con LA $<24 \mathrm{~mm}(\mathrm{r}=0,391 ; \mathrm{p}<$ 0,001 ), siendo nula en el caso de ojos con LA $\geq 24$

\begin{abstract}
Purpose: To report the relationship between different optical ocular components and the influence of axial length on emmetropization.

Methods: 109 young university students, divided into five groups, were enrolled in this study: emmetropes, hyperopes, low myopes, moderate myopes and high myopes. Intraocular parameters and topographic corneal analyses were performed by ultrasonography and videokeratoscopy respectively.

Results: Anterior chamber depth and axial length were found to correlate significantly in eyes with axial lengths less than $24 \mathrm{~mm}(\mathrm{r}=0.441 ; \mathrm{p}<0.001)$ However this correlation was not found in eyes with longer axial lengths $(r=0.098 ; p=0.527)$. Lens thickness showed an inverse correlation with axial length for shorter eyes $(r=0.391 ; p<0.001)$, whereas any correlation in longer eyes was associated with moderate to high levels of myopia. Anterior corneal curvature only correlated, although weakly,
\end{abstract}

\footnotetext{
Recibido: 27/7/06. Aceptado: 8/4/08.

1 Doctora en Farmacia. Escuela Universitaria de Óptica y Optometría. Universidad Santiago de Compostela. Santiago de Compostela. España.

2 Diplomado en Óptica y Optometría. Departamento de Física (Optometría). Universidad do Minho. Braga. Portugal.

3 Diplomado en Óptica y Optometría. Escuela Universitaria de Óptica y Optometría. Universidad Santiago de Compostela. Santiago de Compostela. España.

Los autores no tienen interés comercial alguno en la fabricación, suministro o distribución de los instrumentos utilizados en este estudio.
}

Correspondencia:

Eva Yebra-Pimentel

Escuela de Óptica y Optometría. Campus Sur

Universidad de Santiago de Compostela

15706 Santiago de Compostela

España

E-mail: eyebra@usc.es 
mm. La curvatura corneal (RC) sólo mostró una débil correlación con la profundidad de la cámara vítrea (PCV) en ojos con LA $<24$ mm ( $\mathrm{r}=0,363$; $\mathrm{p}$ $<0,003)$.

Conclusión: Los parámetros ópticos del ojo humano se relacionan de distinta forma según el tamaño del ojo, encontrándose en los ojos más miopes una ausencia de coordinación entre los componentes ópticos oculares, que según los resultados de este estudio, es a partir de 2,00 D.

Palabras clave: Error refractivo, componentes ópticos oculares, longitud axial, ametropía, emetropización. with vitreous chamber depth for shorter eyes $(\mathrm{r}=$ 0.363; $\mathrm{p}<0.003)$.

Conclusion: Differences in optical parameters of the human eye displayed different associations in longer eyes. A lack of correlation was seen between ocular components in eyes with higher myopia, corresponding to myopia in excess of $2.00 \mathrm{D}$ (Arch Soc Esp Oftalmol 2008; 83: 307-316).

Key words: Refractive error, axial length, ocular components, ametropia, emmetropization.

\section{INTRODUCCIÓN}

Existen numerosos estudios (longitudinales, trasversales y epidemiológicos) que tratan de ampliar los conocimientos sobre el desarrollo y progresión de los errores refractivos, centrándose en la relación entre los componentes oculares, la topografía corneal y en su papel en el desarrollo de la ametropía (1-4). De la misma forma, también han cobrado interés las implicaciones en cirugía refractiva (5-7) o en la calidad óptica del ojo (8). Entre los componentes oculares que pueden influir en el error refractivo ( $\mathrm{Rx}$ ) se encuentran: el radio de curvatura corneal anterior ( $\mathrm{RC})$, la profundidad de la cámara anterior (PCA), el espesor del cristalino (EC), la profundidad de la cámara vítrea (PCV) y la longitud axial (LA).

Es conocida la correlación directa que existe entre la PCA y el diámetro cornal medio, de manera que ojos miopes serían ojos más grandes, con diámetros corneales mayores y cámara anterior más profunda (6). Stone y col (9), investigando en pollos, consideraron que la superficie corneal podría ser una variable útil para evaluar el crecimiento del segmento anterior durante el crecimiento del ojo (9), y un parámetro más adecuado que las medidas convencionales de PCA o RC.

La medida de la PCA proporciona información sobre el crecimiento anterior y ecuatorial del ojo. Por otro lado, la PCV y la LA son considerados como los índices más representativos del crecimiento del segmento posterior, además de factores principales en la progresión de la miopía $(3,10)$. Sin embargo, todavía no se demostrado si existe algún umbral para la correlación entre parámetros sagita- les y axiales responsables de la aparición de miopía alta o moderada.

Estudios previos en pollos han demostrado la disociación de los mecanismos responsables del crecimiento del segmento anterior y de la cámara vítrea. Por ejemplo, bajo ciertas condiciones de iluminación se ha visto que la PCA disminuye, pero aumenta la PCV $(11,12)$.

Hosny y col (6) encontraron una correlación entre la PCA y la LA en humanos, así como entre la PCA y el grado de miopía, observándose también un aumento de la PCA con la miopía. Sin embargo, con la edad se observó una correlación inversa. Un estudio más detallado realizado por estos autores reveló que la PCA parece aumentar con la LA hasta un cierto grado, a partir del cual un incremento en la dirección posterior no implicaría necesariamente un aumento de la PCA, es decir, en la dirección ecuatorial. Se observó que, para una población con una LA entre 19 y 33,2 mm, la correlación entre PCA y LA que existía en ojos más pequeños, se perdía cuando la LA era mayor o igual que $26 \mathrm{~mm}$ (6).

La asfericidad corneal y sus relaciones con los restantes componentes ópticos oculares han sido estudiados recientemente por los presentes autores (13). Existen otros descriptores corneales que representan la forma asférica de la córnea (14).

El objetivo de este estudio es evaluar la relación entre los componentes ópticos oculares y su potencial influencia en el estado refractivo del ojo.

Para ello se determinarán los componentes ópticos oculares de una muestra de sujetos, que incluirá los datos corneales (radio corneal y asfericidad) y los valores biométricos (LA, PCA, PCV y EC). 


\section{SUJETOS, MATERIAL Y MÉTODO}

\section{Muestra}

Después de obtener el consentimiento informado se examinó el OD de 109 pacientes caucásicos (67 mujeres, 42 hombres) de edades comprendidas entre 15 y 35 años (22,27; DE 3,24 años).

Sólo fueron incluidos en el estudio aquellos sujetos que alcanzaban con su mejor corrección una agudeza visual (AV) igual o mejor que 20/20. No fueron incluidos aquellos que presentaban patología corneal examinados con lámpara de hendidura ni patología del polo posterior mediante oftalmoscopia directa. Además ningún sujeto presentaba patología corneal, ni estaba tomando medicación ocular o sistémica que pudiese producir cambios en la córnea o en el cristalino. Previamente a la realización del estudio, todos los procedimientos fueron aprobados por el Comité de Bioética para la Investigación con Humanos de la Universidad de Santiago de Compostela.

El estado refractivo de la muestra obtenido mediante refracción ciclopléjica mostró una esfera equivalente media de -1,66, DE 2,52 D (rango de +3,00 D a $-11,00 \mathrm{D})$. El astigmatismo era menor de 1,50 D.

La muestra se dividió, con un número similar de sujetos, edad y sexo, en 5 grupos refractivos siguiendo el criterio establecido por Carney y col. (2): «emétropes» [equivalente esférico (SE), -0,25 a $+0,25 \mathrm{D}], \mathrm{n}=30$; «hipermétropes» $(\mathrm{SE} \geq+0,50 \mathrm{y} \leq$ $+3,25 \mathrm{D}), \mathrm{n}=20$; «miopes bajos» ( $\mathrm{SE} \geq-0,50 \mathrm{y} \leq-$ 2,00 D), $\mathrm{n}=20$; «miopes moderados» $(\mathrm{SE}>-2,00 \mathrm{y}$ $\leq-4,00 \mathrm{D}), \mathrm{n}=20 \mathrm{y}$ «miopes altos» $(\mathrm{SE}>-4,00 \mathrm{D})$, $\mathrm{n}=19$.

\section{Procedimientos}

Los valores de curvatura corneal anterior (RC) y asfericidad (Q) fueron determinados mediante videoqueratoscopía (EyeSys Laboratories, Salt Lake City, UT) entre las 14:00 y 17:00 h. con el fin de evitar el sesgo inducido por las variaciones diurnas de la curvatura corneal. El RC se obtuvo del mapa queratométrico, mientras que $\mathrm{Q}$ se calculó en la zona de 4,5 mm usando el Holladay Diagnostic Summary (4). En ambos casos el valor final se corresponde al promedio de tres medidas. Previo a cada sesión el videoqueratoscopio se calibró con un set de esferas de radio conocido. Posteriormente, y tras la instilación de una gota de tetracaína al 0,5\%, se determinaron los parámetros ópticos oculares PCA, EC, PCV y LA con el biómetro ultrasónico Ophthasonic ${ }^{\circledR}$ A-scan/Pachometer III (Teknar Inc., St. Louis, MO), realizando cinco lecturas en cada caso y promediándolas. Sólo se aceptaron si la DE de las cinco medidas era igual o inferior que 0,1 $\mathrm{mm}$, en caso contrario se procedió a repetir las medidas. Todas las medidas se realizaron bajo cicloplejia con el fin de eliminar la influencia de la acomodación en el EC y en otras estructuras potencialmente influenciables como la PCA y PCV (15).

\section{Análisis estadístico}

Para el análisis de los datos se utilizó el software de estadística SPSS v. 12,0.1 para Windows (SPSS Inc., Chicago, USA). Una vez verificada la igualdad de varianzas correspondiente a los cinco grupos refractivos con el test de Levene, se aplicó un test ANOVA entre grupos para comparar el valor medio obtenido en cada caso. La relación entre los diferentes parámetros oculares $(\mathrm{Q}, \mathrm{RC}, \mathrm{PCA}, \mathrm{EC}, \mathrm{PCV}$ y LA) se estudió mediante un análisis de regresión lineal, con un total de veintiuna combinaciones posibles y a un nivel de significación de $\mathrm{p}<0,0024$.

\section{RESULTADOS}

En la tabla I se presentan los valores refractivos, topográficos y biométricos correspondientes a cada grupo refractivo. Se aprecia que el EC, PCV y LA varían con el estado refractivo. De esta forma, se encontró que la LA y el PCV eran mayores en miopes que hipermétropes, incrementándose con el grado de miopía. Sin embargo el EC no mostró una relación estadísticamente significativa, aunque se observó una tendencia a aumentar con el grado de miopía. La PCA no mostró diferencias estadísticamente significativas entre los distintos grupos refractivos.

En la tabla II se indica la correlación entre los distintos parámetros oculares. La PCA mostró una correlación estadísticamente significativa con el EC y la LA, pero no con la PCV. En la figura 1 se ilustra la recta de regresión entre EC y PCA donde se puede apreciar la relación negativa entre ambos.

En la figura 2 se presenta un modelo cuadrático de regresión que describe la relación entre PCA y 
Tabla I. Estadística descriptiva para las medidas biométricas y topográficas oculares. Se muestra la correspondiente distribución por edad y sexo para los cinco grupos refractivos

\begin{tabular}{|c|c|c|c|c|c|c|c|c|c|c|c|}
\hline & & \multicolumn{2}{|c|}{$\begin{array}{l}\text { Hipermétropes } \\
(n=20)\end{array}$} & \multicolumn{2}{|c|}{$\begin{array}{l}\text { Emétropes } \\
\qquad(n=30)\end{array}$} & \multicolumn{2}{|c|}{$\begin{array}{l}\text { Miopes bajos } \\
\quad(\mathrm{n}=20)\end{array}$} & \multicolumn{2}{|c|}{$\begin{array}{l}\text { Miopes moderados } \\
\qquad(\mathrm{n}=20)\end{array}$} & \multicolumn{2}{|c|}{$\begin{array}{l}\text { Miopes altos } \\
(\mathrm{n}=19)\end{array}$} \\
\hline \multicolumn{2}{|c|}{$\begin{array}{l}\text { Hombres/Mujeres } \\
\text { Edad (años) }\end{array}$} & \multicolumn{2}{|c|}{$\begin{array}{c}9 / 11 \\
22,30 \mathrm{DE} 3,33\end{array}$} & \multicolumn{2}{|c|}{$\begin{array}{c}13 / 17 \\
21,70 \text { DE 2,71 }\end{array}$} & \multicolumn{2}{|c|}{$\begin{array}{c}6 / 14 \\
22,20 \text { DE 2,57 }\end{array}$} & \multicolumn{2}{|c|}{$\begin{array}{c}6 / 14 \\
22,50 \text { DE 2,86 }\end{array}$} & \multicolumn{2}{|c|}{$\begin{array}{c}8 / 11 \\
22,95 \mathrm{DE} 4,7 \mathrm{C}\end{array}$} \\
\hline & Sig. p* & Media & DS & Media & DS & Media & DS & Media & DS & Media & DS \\
\hline $\mathrm{Rx}$ & $<0,001$ & 0,99 & 0,72 & $-0,05$ & 0,15 & $-1,33$ & 0,56 & $-3,10$ & 0,50 & $-5,83$ & 1,95 \\
\hline $\mathrm{RC}$ & N.S & 7,87 & 0,21 & 7,81 & 0,22 & 7,82 & 0,23 & 7,86 & 0,27 & 7,82 & 0,25 \\
\hline Q & N.S & $-0,20$ & 0,08 & $-0,23$ & 0,07 & $-0,22$ & 0,09 & $-0,26$ & 0,10 & $-0,4$ & 0,06 \\
\hline PCA & N.S & 3,42 & 0,28 & 3,61 & 0,30 & 3,56 & 0,27 & 3,66 & 0,32 & 3,52 & 0,30 \\
\hline $\mathrm{EC}$ & 0,003 & 3,66 & 0,23 & 3,57 & 0,17 & 3,56 & 0,18 & 3,64 & 0,23 & 3,81 & 0,26 \\
\hline PCV & $<0,001$ & 15,72 & 0,80 & 16,16 & 0,60 & 16,43 & 0,74 & 17,02 & 0,77 & 17,92 & 1,10 \\
\hline LA & $<0,001$ & 22,80 & 0,85 & 23,34 & 0,65 & 2,55 & 0,75 & 24,32 & 0,9 & 25,25 & 1,10 \\
\hline
\end{tabular}

Significación estadística para el test ANOVA entre grupos refractivos.

Rx: error refractivo; RC: radio de curvatura corneal anterior; Q: asfericidad; PCA: profundidad de la cámara anterior; EC: espesor del cristalino; PCV: profundidad de la cámara vítrea; LA: longitud axial. Se muestra la correspondiente distribución por edad y sexo para los cinco grupos refractivos.

LA. Esta representación se adapta a la totalidad de los datos $\left(r^{2}=0,188 ; p<0,001\right)$ de un modo mas fiable que el modelo lineal $\left(\mathrm{r}^{2}=0,156 ; \mathrm{p}<0,001\right)$, evidenciando una correlación positiva hasta un punto de inflexión situado entre 23 y $25 \mathrm{~mm}$ de LA. Por este motivo se decidió establecer el límite en $24 \mathrm{~mm}$ para los análisis sucesivos, dividiendo la muestra en dos subgrupos: aquellos con una LA menor a 24 $\mathrm{mm}(\mathrm{n}=65)$ y aquellos con una LA igual o mayor a $24 \mathrm{~mm}(\mathrm{n}=44)$.

En la figura 3 se muestra la relación entre PCA y LA para ambos subgrupos por separado. En este gráfico se puede apreciar la pérdida de correlación entre ambos parámetros en ojos de mayor tamaño
(LA $\geq 24 \mathrm{~mm}$ ), en los que se observa un aumento de la LA sin un aumento significativo de la PCA.

En la figura 4 se presenta la relación entre la $\mathrm{Rx}$ y la LA, donde el límite establecido (LA igual a 24 $\mathrm{mm}$ ) se corresponde con un error Rx de -2,00 D. Por lo tanto, en términos refractivos, valores mayores de $-2,00 \mathrm{D}$ se pueden aplicar a miopía moderada y alta y valores menores que $-2,00 \mathrm{D}$ a ojos con otras condiciones refractivas. La estadística descriptiva para ambos grupos se muestra en la tabla III. Ojos más grandes presentan valores mayores en las medidas biométricas, así como corneas más prolatas y lecturas queratométricas centrales más planas.

Tabla II. Resultado del análisis estadístico multidimensional aplicado a toda la muestra (n=109) donde se indica el coeficiente de correlación «r» y el nivel de significación estadística «p» para todas las variables biométricas y topográficas

\begin{tabular}{lccccccc}
\hline Parámetros Oculares & RC & Q & PCA & EC & PCV & LA & Rx \\
\hline RC & & $\mathrm{p}=0,025$ & $\mathrm{p}=0,767$ & $\mathrm{p}=0,836$ & $\mathbf{p}<\mathbf{0 , 0 0 1}$ & $\mathbf{p}<\mathbf{0 , 0 0 1}$ & $\mathrm{p}=0,726$ \\
$\mathrm{Q}$ & $\mathrm{r}=0,214$ & & $\mathrm{p}=0,853$ & $\mathrm{p}=0,535$ & $\mathbf{p}=\mathbf{0 , 0 0 3} *$ & $\mathrm{p}=0,007$ & $\mathrm{p}=0,075$ \\
PCA & $\mathrm{r}=0,029$ & $\mathrm{r}=0,018$ & & $\mathbf{p}<\mathbf{0 , 0 0 1}$ & $\mathrm{p}=0,015$ & $\mathrm{p}<0,001$ & $\mathrm{p}=0,338$ \\
EC & $\mathrm{r}=0,020$ & $\mathrm{r}=0,060$ & $\mathrm{r}=0,409$ & & $\mathrm{p}=0,435$ & $\mathrm{p}=0,848$ & $\mathrm{p}=0,026$ \\
PCV & $\mathrm{r}=0,372$ & $\mathrm{r}=0,287$ & $\mathrm{r}=0,233$ & $\mathrm{r}=0,076$ & & $\mathbf{p}<\mathbf{0 , 0 0 1}$ & $\mathbf{p}<\mathbf{0 , 0 0 1}$ \\
LA & $\mathrm{r}=0,354$ & $\mathrm{r}=0,258$ & $\mathrm{r}=0,394$ & $\mathrm{r}=0,019$ & $\mathrm{r}=0,969$ & & $\mathbf{p}<\mathbf{0 , 0 0 1}$ \\
Rx & $\mathrm{r}=0,034$ & $\mathrm{r}=0,171$ & $\mathrm{r}=0,093$ & $\mathrm{r}=0,214$ & $\mathrm{r}=0,742$ & $\mathrm{r}=0,750$ & \\
\hline \hline
\end{tabular}

Sobre las celdas sombreadas se representa la significación estadística. Por debajo de las celdas sombreadas se representan los coeficientes de correlación. Los valores presentados en negrita identifican aquellas relaciones estadísticamente significativas de acuerdo al criterio establecido.

* La relación entre PCV y Q solo alcanza significación estadística una vez que el nivel de significación se establece en p = 0,0024.

Rx: error refractivo; RC: radio de curvatura corneal anterior, Q: asfericidad, PCA: profundidad de la cámara anterior; EC: espesor del cristalino; PCV: profundidad de la cámara vítrea; LA: longitud axial. 


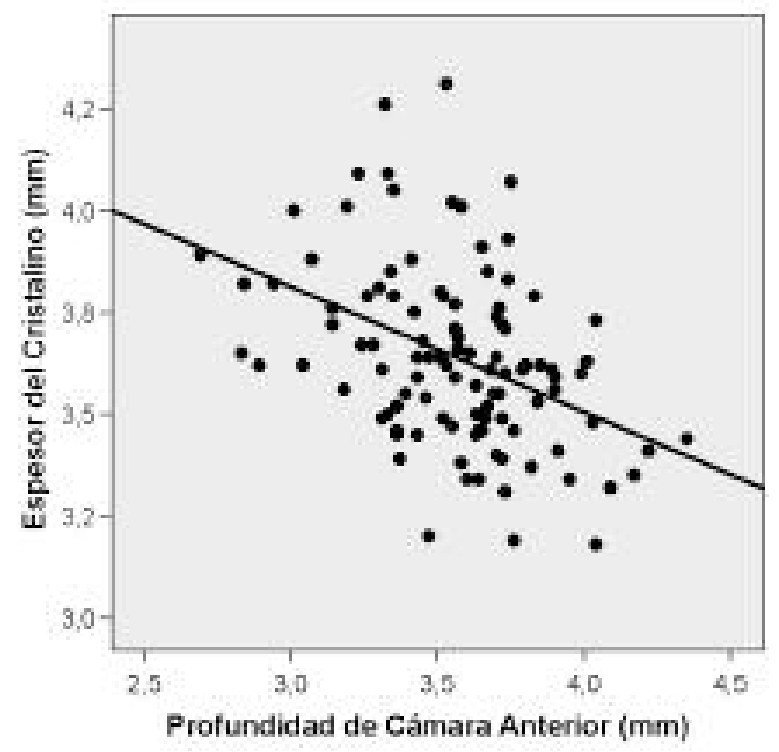

Fig. 1: Regresión lineal entre la profundidad de la cámara anterior (PCA) y espesor del cristalino (EC).

En la tabla IV se presenta la correlación entre los distintos parámetros oculares dividiendo a la muestra en los dos subgrupos según la LA, tal como se describía en la tabla III. Se observa que, con respecto a la tabla II (muestra global), la correlación entre los parámetros aumenta en ambos subgrupos.

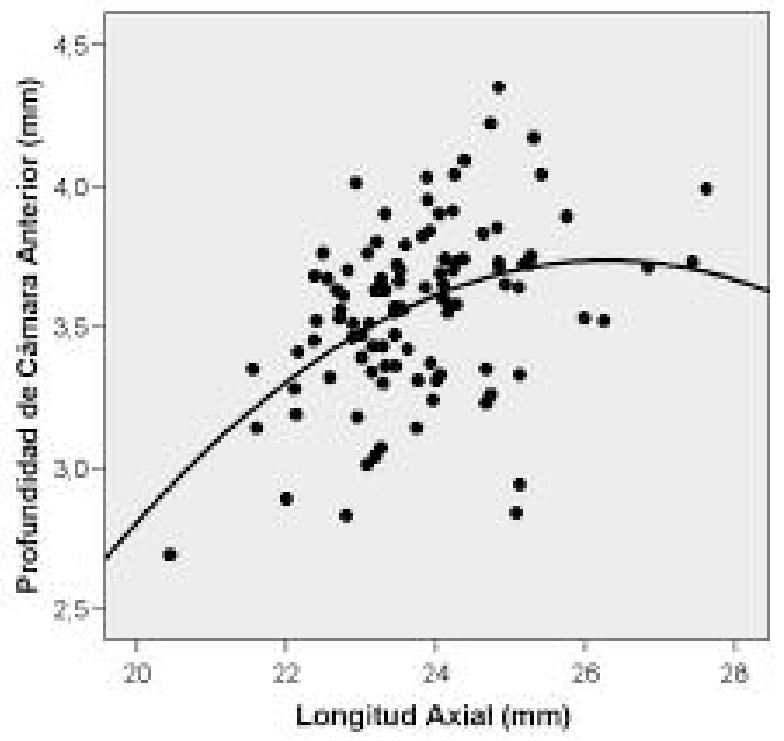

Fig. 2: Modelo cuadrático de regresión entre la longitud axial (LA) y la profundidad de la cámara anterior (PCA).

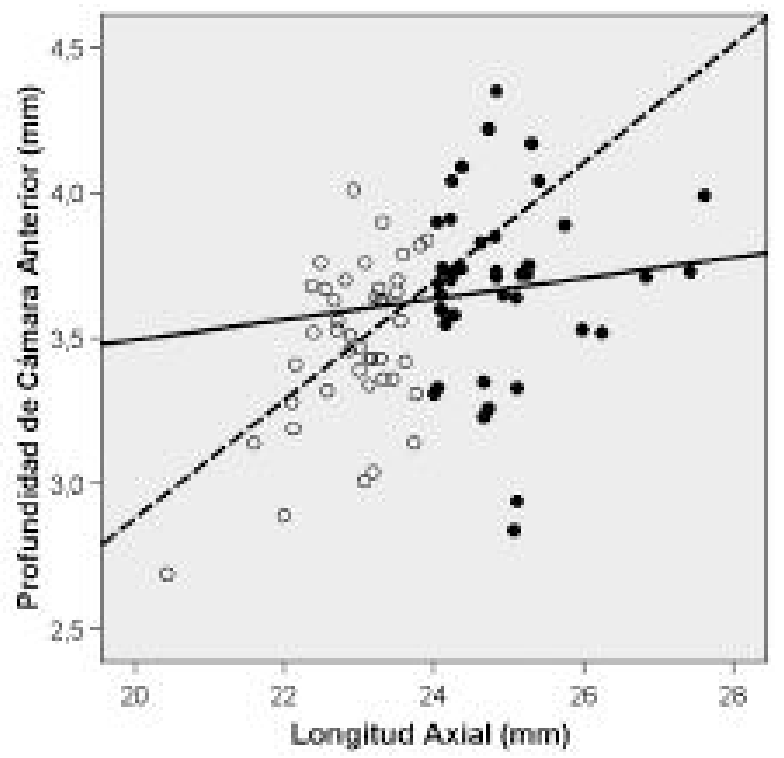

Fig. 3: Regresión lineal mostrando la asociación entre la profundidad de cámara anterior (PCA) y la longitud axial (LA) para ojos con menor LA $(<24 \mathrm{~mm}-$ cuadros negros y línea continua) y ojos con mayor LA ( $\geq 24 \mathrm{~mm}$ - cuadros blancos y línea discontinua).

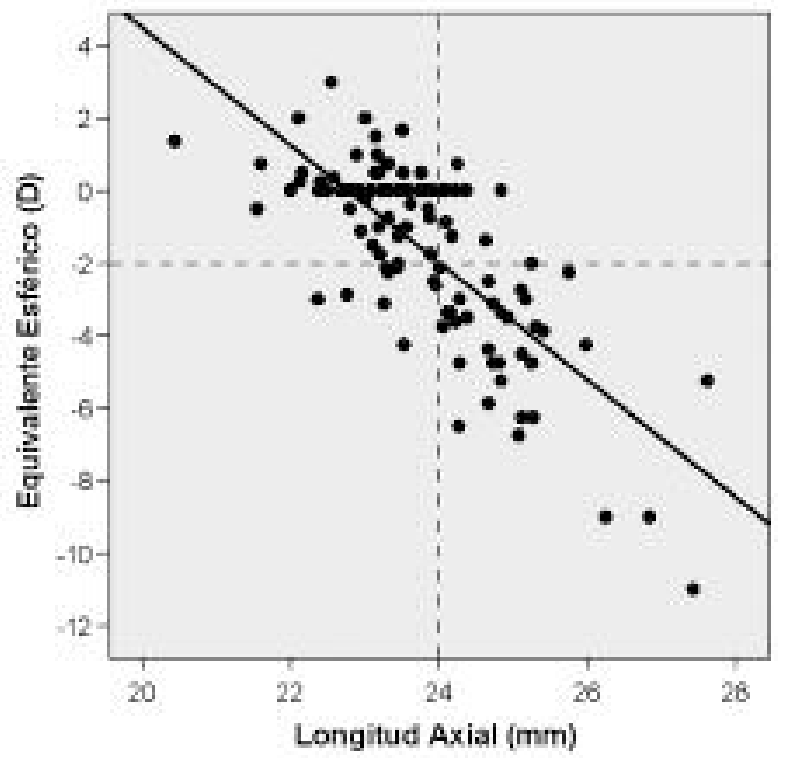

Fig. 4: Representación de la correlación entre el valor del error refractivo (equivalente esférico) ( $R x)$ y la longitud axial (LA). Las líneas de referencia dividen la muestra en ojos con LA menor o mayor de $24 \mathrm{~mm}$ y/o Rx superior o inferior a -2,00 dioptrías. Se ha podido calcular que el $75 \%$ (34/44) de los ojos con LA $\geq 24 \mathrm{~mm}$ tienen miopía superior $a \geq-2,00$ D y que el $86 \%$ (56/65) de los ojos con LA $<24$ mm pertenecen a los restantes grupos (miopía menor de -2,00, emetropía o hipermetropía). 
Tabla III. Estadística descriptiva de los parámetros oculares investigados en este estudio para ojos con mayor longitud axial $(\geq 24 \mathrm{~mm})$ y menor longitud axial $(<24 \mathrm{~mm})$

\begin{tabular}{|c|c|c|c|c|c|c|c|c|}
\hline \multirow[b]{2}{*}{ Parámetros Oculares } & \multicolumn{3}{|c|}{$\mathrm{LA}<24 \mathrm{~mm}(\mathrm{n}=65)$} & \multicolumn{3}{|c|}{$\mathrm{LA} \geq 24 \mathrm{~mm}(\mathrm{n}=44)$} & \multicolumn{2}{|c|}{ Total } \\
\hline & Media & DS & Rango & Media & DS & Rango & Media & DS \\
\hline $\mathrm{RC}$ & 7,79 & 0,19 & {$[7,36$ a 8,21$]$} & 7,91 & 0,27 & {$[7,33$ a 8,43$]$} & 7,84 & 0,23 \\
\hline Q & $-0,21$ & 0,08 & {$[-0,03$ a $-0,41]$} & $-0,26$ & 0,07 & {$[-0,14$ a $-0,42]$} & $-0,23$ & 0,08 \\
\hline PCA & 3,48 & 0,27 & {$[2,69$ a 4,03$]$} & 3,67 & 0,31 & {$[2,84$ a 4,35$]$} & 3,56 & 0,30 \\
\hline $\mathrm{EC}$ & 3,62 & 0,18 & {$[3,19$ a 4,26$]$} & 3,67 & 0,27 & {$[3,18$ a 4,31$]$} & 3,64 & 0,23 \\
\hline PCV & 15,94 & 0,65 & {$[13,85$ a 17,16$]$} & 17,55 & 0,85 & {$[16,44$ a 20,09$]$} & 16,59 & 1,07 \\
\hline LA & 23,05 & 0,65 & {$[20,43$ a 23,95$]$} & 24,89 & 0,86 & {$[24,27$ a 27,61$]$} & 23,79 & 1,16 \\
\hline
\end{tabular}

En cada grupo se muestra el error refractivo (Rx), el radio de curvatura corneal anterior (RC), la asfericidad (Q), la profundidad de la cámara anterior (PCA), el espesor del cristalino (EC), la profundidad de la cámara vítrea (PCV) y longitud axial (LA).

Se encontró una relación estadísticamente significativa entre la PCA y el EC con la LA en el subgrupo con LA $<24 \mathrm{~mm}$. Contrariamente, no se encontró esta relación para ojos con LA > $24 \mathrm{~mm}$. Una relación similar se observó entre el RC y el EC con la PCV. La relación entre PCA y EC también resultó significativa para ambos subgrupos. Asimismo, se observó un comportamiento similar entre LA y PCV. En contraste, la relación entre PCV y LA con el error refractivo es estadísticamente significativa para ojos con LA $\geq 24 \mathrm{~mm}$ y no para ojos con LA < $24 \mathrm{~mm}$.

Por último, existe una correlación estadísticamente significativa entre Q y PCV, lo que implica que ojos con mayor PCV tienden a córneas más prolatas (fig. 5). En cuanto al RC, se observa una relación estadísticamente significativa con la PCV (figura 6), de manera que ojos con mayor crecimiento posterior presentan una curvatura corneal central más plana, pero no se observó esta relación entre el RC y el Rx.

El Rx presentó una correlación estadísticamente significativa solamente con la LA y la PCV en la muestra global (tabla II) y en el subgrupo de LA $\geq 24 \mathrm{~mm}$ (tabla IV).

\section{DISCUSIÓN}

En los últimos años ha habido gran interés en conocer, de manera precisa, las dimensiones del ojo humano.

Tabla IV. Resultados del análisis estadístico multidimensional para ojos con mayor longitud axial $(\leq 24 \mathrm{~mm})$ y con menor longitud axial $(<24 \mathrm{~mm})$. Se muestra el coeficiente de correlación «r» y el nivel de significación estadística «p» entre los parámetros estudiados

\begin{tabular}{lccccccc}
\hline Parámetros Oculares & RC & Q & PCA & EC & PCV & LA & Rx \\
\hline RC & & $\mathrm{r}=0,226$ & $\mathrm{r}=0,027$ & $\mathrm{r}=0,130$ & $\mathrm{r}=0,236$ & $\mathrm{r}=0,202$ & $\mathrm{r}=0,303$ \\
& & $\mathrm{p}=0,141$ & $\mathrm{p}=0,863$ & $\mathrm{p}=0,399$ & $\mathrm{p}=0,123$ & $\mathrm{p}=0,189$ & $\mathrm{p}=0,045$ \\
$\mathrm{Q}$ & $\mathrm{r}=0,091$ & & $\mathrm{r}=0,103$ & $\mathrm{r}=0,276$ & $\mathrm{r}=0,088$ & $\mathrm{r}=0,036$ & $\mathrm{r}=0,103$ \\
& $\mathrm{p}=0,472$ & & $\mathrm{p}=0,508$ & $\mathrm{p}=0,069$ & $\mathrm{p}=0,569$ & $\mathrm{p}=0,817$ & $\mathrm{p}=0,507$ \\
PCA & $\mathrm{r}=0,128$ & $\mathrm{r}=0,217$ & & $\mathbf{r}=\mathbf{0 , 5 1 3}$ & $\mathrm{r}=0,101$ & $\mathrm{r}=0,098$ & $\mathrm{r}=0,276$ \\
& $\mathrm{p}=0,310$ & $\mathrm{p}=0,083$ & & $\mathbf{p}<\mathbf{0 , 0 0 1}$ & $\mathrm{p}=0,514$ & $\mathrm{p}=0,527$ & $\mathrm{p}=0,069$ \\
EC & $\mathrm{r}=0,161$ & $\mathrm{r}=0,051$ & $\mathbf{r}=\mathbf{0 , 4 2 6}$ & & $\mathrm{r}=0,036$ & $\mathrm{r}=0,099$ & $\mathrm{r}=0,300$ \\
& $\mathrm{p}=0,201$ & $\mathrm{p}=0,684$ & $\mathbf{p}<\mathbf{0 , 0 0 1}$ & & $\mathrm{p}=0,815$ & $\mathrm{p}=0,523$ & $\mathrm{p}=0,048$ \\
PCV & $\mathbf{r}=\mathbf{0 , 3 6 3}$ & $\mathrm{r}=0,088$ & $\mathrm{r}=0,141$ & $\mathrm{r}=0,496$ & & $\mathbf{r}=\mathbf{0 , 9 4 2}$ & $\mathbf{r}=\mathbf{0 , 6 6 2}$ \\
& $\mathbf{p}=\mathbf{0 , 0 0 3} *$ & $\mathrm{p}=0,485$ & $\mathrm{p}=0,261$ & $\mathrm{p}<0,001$ & & $\mathbf{p}<\mathbf{0 , 0 0 1}$ & $\mathbf{p}<\mathbf{0 , 0 0 1}$ \\
LA & $\mathrm{r}=0,357$ & $\mathrm{r}=0,012$ & $\mathbf{r}=\mathbf{0 , 4 4 1}$ & $\mathbf{r}=\mathbf{0 , 9 1}$ & $\mathbf{r}=\mathbf{0 , 9 2 2}$ & & $\mathbf{r}=\mathbf{0 , 6 5 1}$ \\
& $\mathrm{p}=0,004$ & $\mathrm{p}=0,925$ & $\mathbf{p}<\mathbf{0 , 0 0 1}$ & $\mathbf{p}=\mathbf{0 , 0 0 1}$ & $\mathbf{p}<\mathbf{0 , 0 0 1}$ & $\mathrm{p}=0,324$ & $\mathbf{p}<\mathbf{0 , 0 0 1}$ \\
Rx & $\mathrm{r}=0,178$ & $\mathrm{r}=0,023$ & $\mathrm{r}=0,057$ & $\mathrm{r}=0,036$ & $\mathrm{r}=0,308$ & $\mathrm{r}=0,324$ & $\mathrm{p}=0,009$ \\
& $\mathrm{p}=0,157$ & $\mathrm{p}=0,854$ & $\mathrm{p}=0,652$ & $\mathrm{p}=0,778$ & $\mathrm{p}=0,012$ & $\mathrm{p}=0,0$ \\
\hline \hline
\end{tabular}

Sobre las celdas sombreadas se presentan los resultados estadísticos para ojos con una LA $\geq 24$ mm, y por debajo los correspondientes a ojos con una LA $<24 \mathrm{~mm}$. Los resultados en negrita identifican las relaciones entre parámetros estadísticamente significativas según el criterio establecido. * La relación entre PCV y RC no alcanza significación estadística una vez que el nivel de significación se establece en p = 0,0024.

Rx: error refractivo; RC: radio de curvatura corneal anterior; Q: asfericidad; PCA: profundidad de la cámara anterior; EC: espesor del cristalino; PCV: profundidad de la cámara vítrea; LA: longitud axial. 


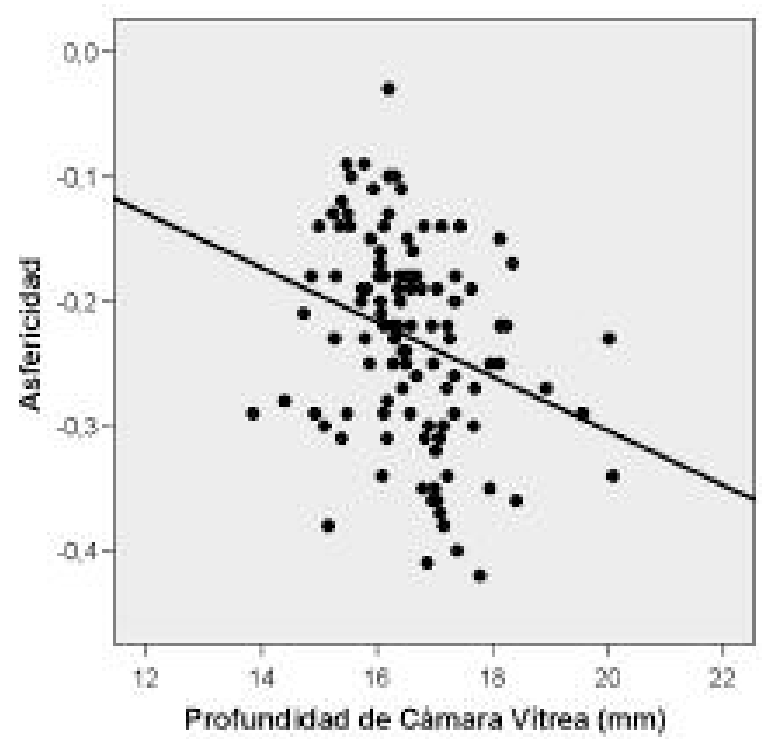

Fig. 5: Regresión lineal entre la asfericidad corneal $(Q)$ y la profundidad de la cámara vítrea (PCV).

Además de estudiar la elongación posterior del globo ocular, sería de gran utilidad conocer qué parámetros reflejan el crecimiento anterior del ojo humano para establecer si una pérdida en el equilibrio entre ambos segmentos podría estar relacionada con la aparición y desarrollo de la ametropía. Estudios previos sugieren que el diámetro o el área de la superficie corneal son indicadores del creci-

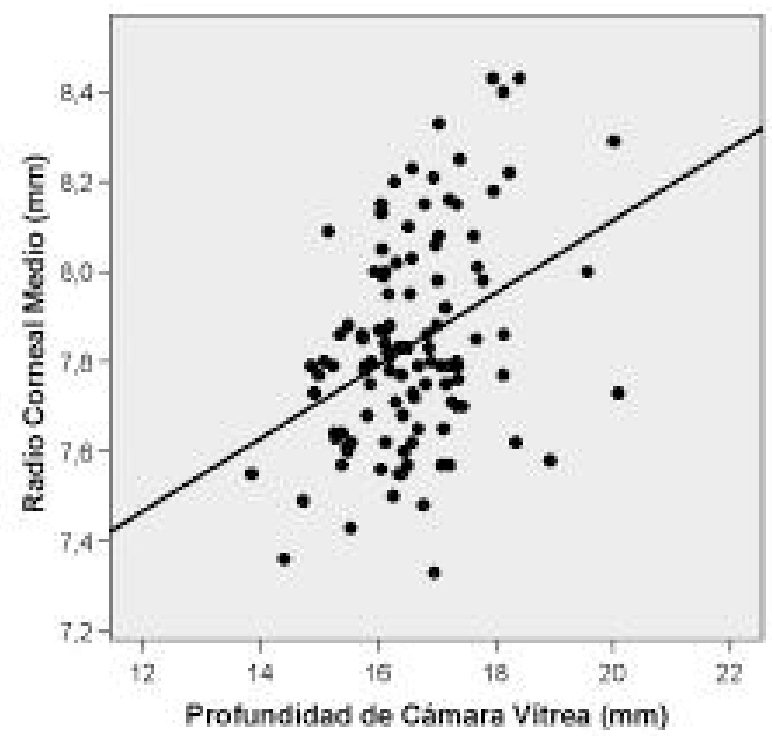

Fig. 6: Regresión lineal entre el radio corneal central medio $(R C)$ y la profundidad de la cámara vítrea $(P C V)$. miento del segmento anterior del ojo (11). Hosny y col (6) describieron la correlación existente entre la PCA y el diámetro corneal medio, por lo que, indirectamente, la PCA podría ser considerada como un índice del crecimiento trasversal y ecuatorial del segmento anterior del ojo.

Los resultados, similares a los de Hosny y col (6), mostraron una correlación estadísticamente significativa entre la PCA y la LA. Sin embargo, después de dividir la muestra en dos subgrupos según la LA, se observó que para el subgrupo con LA $<24$ $\mathrm{mm}$ presentaba una correlación estadísticamente significativa entre estas dos variables mientras que en el subgrupo de LA $\geq 24 \mathrm{~mm}$ no presentaba esta correlación.

La elección de 24 mm como el límite en la LA se realizó considerando por un lado el valor medio comúnmente aceptado de LA del ojo humano, y por otro lado por ser el punto medio del intervalo entre 23 y $25 \mathrm{~mm}$, donde se produce el cambio de inflexión de la función (fig. 2). Con esta elección cada subgrupo queda formado por un número estadísticamente representativo. Además, en el recta de regresión entre la LA y el error refractivo de la muestra (fig. 4), puede verse que la LA igual a $24 \mathrm{~mm}$ se corresponde con un error refractivo de -2,00 D. Esta elección es clínicamente aceptable, puesto que el 75\% (34/44) de los ojos con una $\mathrm{AL} \geq 24 \mathrm{~mm}$ presentaban una miopía mayor de -2,00 D (miopía moderada- alta), mientras que el 86\% (56/65) de los ojos con una $\mathrm{AL}<24 \mathrm{~mm}$ formaban los restantes grados refractivos (miopía < 2,00 D, emetropía e hipermetropía).

Los resultados encontrados en este estudio también sugieren que la profundidad de la cámara anterior aumenta con la LA hasta un cierto valor, a partir del cual la elongación del ojo no acompañaría un incremento de la PCA. Este hallazgo apoya la teoría según la cual el crecimiento del ojo es un proceso coordinado y armónico tanto en la dirección ecuatorial como posterior, garantizando el aumento equilibrado de las cámaras anterior y vítrea hasta un cierto nivel, que de superarse, se perdería este equilibrio y aparecería la ametropía. Sin embargo, hay que considerar que el intervalo de LA obtenido en este estudio es relativamente pequeño, desde 20 a $28 \mathrm{~mm}$, ya que en otros trabajos el rango de LA se extendía hasta $33 \mathrm{~mm}$ (6). Por lo tanto, sería necesario un mayor número de casos de miopía elevada para garantizar resultados más concluyentes.

Existen estudios que muestran un importante papel del cristalino en el mecanismo de emetropi- 
zación, sin embargo, se ha prestado poca atención a su relación con la cámara anterior y vítrea. Garner y col (15), en un estudio trasversal realizado con niños tibetanos, encontraron que el equilibrio entre la disminución de la potencia del cristalino y el incremento de la longitud vítrea era el principal factor que mantenía la tendencia hacia la emetropía (15). En otro trabajo longitudinal retrospectivo, en donde se estudiaba la miopía al principio de la edad adulta, se determinó que la principal causa del desarrollo y progresión de la miopía era la elongación vítrea (16).

Garner y Yap (17) demostraron que la PCA decrece con la acomodación como resultado del engrosamiento (aumento de espesor) del cristalino. En la actualidad y gracias a la precisión que permiten las técnicas de medida del segmento anterior del ojo, tal como la interferometría coherente parcial, se ha demostrado que durante la acomodación la cara anterior del cristalino se adelanta aproximadamente 200 micras, mientras que la posterior se desplaza hacia atrás unas 70 micras (18). Como resultado, y asumiendo que las otras superficies, córnea y retina, permanecen invariables, se produce una disminución, de la misma magnitud, en la profundidad de las cámara anterior y posterior respectivamente.

Los resultados encontrados en este estudio sugieren que un incremento en el EC está asociado a una disminución en la PCA, tanto en el caso de errores refractivos menores y mayores de $-2,00 \mathrm{D}$. Una resultado similar se encontró al relacionar la $\mathrm{PCV}$ con el EC en errores refractivos menores de $-2,00$ D, sin embargo, para miopías mayores de $-2,00 \mathrm{D}$ no se encontró esta correlación. Este hallazgo concuerda con la teoría de que la miopía elevada es el resultado de un desequilibrio en el proceso de crecimiento en las direcciones posterior (PCV) y ecuatorial (PCA), por lo tanto el papel del cristalino en el proceso de emetropización sería mínimo. En miopías moderadas y elevadas, se observa un incremento del EC que conlleva a una reducción de la PCA, pero no de la PCV, ya que el crecimiento excesivo del polo posterior enmascara la pequeña aportación del cristalino.

En este estudio no se encontró correlación entre la asfericidad corneal y el error refractivo, lo que concuerda con los resultados de estudios anteriores (13). Sin embargo, investigaciones posteriores sugieren que miopes jóvenes con córneas más prolatas tenderían a desarrollar mayores grados de miopía juvenil (4). En el presente estudio se encon- tró una correlación estadísticamente significativa entre Q y PCV, de manera que ojos con mayor LA tienen valores de asfericidad más negativos.

En conclusión, se presenta un detallado análisis estadístico sobre las interrelaciones existentes entre los componentes ópticos oculares, analizando de manera separada los ojos con mayor y menor LA.

Se observa que la presencia de desequilibrios entre parámetros oculares está directamente relacionada con la aparición y desarrollo de la ametropía. Cabe destacar que en ojos con LA $<24$ mm existía una correlación entre la PCV y RC, así como entre LA con PCA y EC, mientras en ojos con $\mathrm{LA} \geq 24$ mm estas correlaciones no se encuentran.

Según los estudios de Sorby y col. (19) el proceso de emetropización se consideraba acertado hasta un error refractivo máximo de 4,00 D, ya que los distintos componentes óptico-oculares mantenían una correlación dirigida a la emetropización, a pesar de persistir algún grado de error refractivo. En este estudio se actualiza este dato hasta un grado máximo de 2,00 D, más próximo a la emetropía.

\section{BIBLIOGRAFÍA}

1. Goss DA, Jackson TW. Clinical findings before the onset of myopia in youth. I. Ocular optical components. Optom Vis Sci 1995; 72: 870-878.

2. Carney LG, Mainstone JC, Henderson BA. Corneal topography and myopia. A cross-sectional study. Invest Ophthalmol Vis Sci 1997; 38: 311-320.

3. Strang NC, Schmid KL, Carney LG. Hyperopia is predominantly axial in nature. Curr Eye Res 1998; 17: 380-383.

4. Horner DG, Soni PS, Vyas N, Himebaugh NL. Longitudinal changes in corneal asphericity in myopia. Optom Vis Sci 2000; 77: 198-203.

5. Guell JL, Velasco F, Roberts C, Sisquella MT, Mahmoud A. Corneal flap thickness and topography changes induced by flap creation during laser in situ keratomileusis. $J$ Cataract Refract Surg 2005; 31: 115-119.

6. Hosny M, Alio JL, Claramonte P, Attia WH, Perez-Santonja JJ. Relationship between anterior chamber depth, refractive state, corneal diameter, and axial length. J Refract Surg 2000; 16: 336-340.

7. Fleming JF. Corneal asphericity and visual function after radial keratotomy. Cornea 1993; 12: 233-240.

8. Llorente L, Barbero S, Cano D, Dorronsoro C, Marcos $S$. Myopic versus hyperopic eyes: axial length, corneal shape and optical aberrations. J Vis 2004; 4: 288-298.

9. Stone RA, Lin T, Sugimoto R, Capehart C, Maguire MG, Schmid GF. Corneal surface area: an index of anterior segment growth. Ophthalmic Physiol Opt. 2001; 21: 286295.

10. Parafita M, Perez MV, Yebra-Pimentel E, Giraldez MJ, Gonzalez. J. Study of the correlations between refractive 
ocular optic components in a young-adult population. Can J Optom 1998; 60: 217-221.

11. Stone RA, Lin T, Desai D, Capehart C. Photoperiod, early post-natal eye growth, and visual deprivation. Vision Res 1995; 35: 1195-1202.

12. Yebra-Pimentel E, Giraldez MJ, Glez-Meijome JM, Cerviño A, García-Resúa C, Parafita MA. Variación de la ratio longitud axial/radio corneal ( $L A / R C)$ con el estado refractivo ocular. relación con los componentes oculares. Arch Soc Esp Oftalmol 2004; 79: 317-324.

13. Yebra-Pimentel E, Gonzalez-Meijome JM, Cervino A, Girález JM, González-Pérez, J, Parafita MA. Asfericidad en corneal en una población de adultos jóvenes. Implicaciones clínicas. Arch Soc Esp Oftalmol 2004; 79: 385-392.

14. Lindsay R, Smith G, Atchison D. Descriptors of corneal shape. Optom Vis Sci 1998; 75: 156-158.
15. Garner LF, Yap MK, Kinnear RF, Frith MJ. Ocular dimensions and refraction in Tibetan children. Optom Vis Sci 1995; 72: 266-271.

16. McBrien NA, Adams DW. A longitudinal investigation of adult-onset and adult-progression of myopia in an occupational group. Refractive and biometric findings. Invest Ophthalmol Vis Sci 1997; 38: 321-333.

17. Garner LF, Yap MK. Changes in ocular dimensions and refraction with accommodation. Ophthalmic Physiol Opt 1997; 17: 12-17.

18. Drexler W, Baumgartner A, Findl O, Hitzenberger $C K$, Fercher AF. Biometric investigation of changes in the anterior eye segment during accommodation. Vision Res 1997; 37: 2789-2800.

19. Grosvenor T. Miopía. In: Grosvenor T. Optometría de atención primaria. Barcelona: MASSON S.A.; 2005: 63-91. 